\title{
Nerve Interactions and Regenerative Processes Occurring in Newt Limbs Fused End-to-End ${ }^{1}$
}

\author{
Bruce M. Carlson, Steven E. Civiletto ${ }^{2}$ and Harry G. Goshgarian \\ Department of Anatomy, University of Michigan, Ann Arbor, Michigan 48104
}

Accepted November 16, 1973

\begin{abstract}
Peripheral nerve interactions and regenerative phenomena were studied in newt forelimbs fused end to end. After simple fusion, one or two spikelike structures regenerated at the plane of fusion in $88 \%$ of the cases. When one of the limbs was denervated at the time of fusion, no regeneration occurred from the plane of fusion. If the limbs were fused and one was amputated at the shoulder more than 10 days after fusion, regeneration from the amputation surface did not occur. When the limbs were reamputated 30 days later, regeneration of left limbs from the proximodistally reversed right limb stumps followed. If one of the limbs was dencrvated at the time of fusion, and amputation was subsequently carried out through the formerly denervated limb, regeneration always took place after the first amputation. On the basis of these results it is postulated that when regenerating nerves of opposite proximodistal polarity meet head-on, the majority of fibers, at least, do not grow into territories occupied by the other nerve. These results have also demonstrated that full limb regeneration can occur at a greater distance from the midline than the end of a normal limb. These experiments also provide a technique for artificially elongating peripheral nerves.
\end{abstract}

\section{INTRODUCTION}

The regeneration of an amphibian limb is a highly polarized process, with a strong proximodistal formative gradient predominating. The regeneration of nerve fibers within an intact limb similarly follows a proximodistal gradient. Under normal circumstances these regenerating structures do not encounter tissues with an opposite proximodistal polarity. The expcriments to be reported here were originally designed to provide background information for a series of experiments on the interaction of regenerating nerve trunks of opposite polarity, but because of their relevance to limb regeneration theory, they were expanded and are being reported separately.

The primary question was how regenerating nerves will interact when a nerve from one limb is directly confronted by a

\footnotetext{
${ }^{1}$ Supported by grants from the Muscular Dystrophy Associations of America and the University of Michigan Cancer Research Institute.

${ }^{2}$ Present address: Baylor College of Medicine, Houston, Texas 77025 .
}

nerve regenerating from the contralateral limb. Will the nerves continue to regenerate according to their respective proximodistal polarities and bypass one another or will there be a mutually exclusive effect when the fibers of one regenerating nerve approach the territory of the other? The experimental model used to investigate this problem was end-to-end fusion of amputated forelimbs in the newt. After this procedure, the fibers regenerating out from the transected nerve trunks of each limb would not be prevented by any structural barrier from traversing the plane of fusion and growing up the contralateral limb, contrary to the prevailing proximodistal gradient of that limb. These background experiments were not designed to provide a direct answer to the main question of nerve interactions, but the results unexpectedly enabled us to propose a solution on the basis of indirect evidence. This was done primarily by observing gross regenerative reactions of limbs. Subsequent work with nerve stains was done to verify some of the major conclusions. 


\section{MATERIALS AND METHODS}

Over 200 adult newts (Triturus viridescens), collected by the late $\mathrm{Mr}$. Lewis Babbitt around Petersham, Massachusetts and by the campers from Camp Morgan, Washington, New Hampshire, were used in these experiments. Experimental animals were kept in groups of 5 in glass bowls at $21^{\circ} \mathrm{C}$. For all operations the newts were narcotized in 1:1000 solutions of MS 222 (Sandoz) or ethyl $m$-aminobenzoate methanesulfonate (Eastman).

The limb-joining operation consisted first of amputating each forelimb midway between the elbow and the wrist and then trimming the protruding bones exposed by retraction of the soft tissues. The dorsoventral and anteroposterior axes of the amputated limbs were aligned, and the limbs were tightly joined by three $7-0$ silk sutures (Ethicon) placed through the skin at roughly equal intervals. After the joining operation all animals were placed in a cold room $\left(4-8^{\circ} \mathrm{C}\right)$ for $6-12$ days to reduce their activity during the early healing period. Despite this, almost all operated animals in preliminary experiments pulled their arms apart when removed from the cold. To prevent postoperative separation of the joined forelimbs in subsequent work, $2 \mathrm{~mm}$ segments of each humerus were removed before the limb-joining operations. In experienced hands this procedure caused minimal damage to the soft tissues of the upper arm, and the limbs remained joined in over $95 \%$ of the cases. The four experimental series included in this report represent variants of the above operation. Specific details of these procedures will be included with the presentation of the results of the experiments. A graphic summary of the experimental design as well as a summary of the results can be seen in Fig. 11.

In all experiments the animals were examined grossly at weekly intervals for the first few months and at approximately monthly intervals thereafter. Almost half of the experimental limbs were subjected to histological analysis at various periods throughout the experiments. Seven-micron sections were stained with Ehrlich's hematoxylin and eosin to study general regenerative events, and $10-12 \mu \mathrm{m}$ sections were stained with Hsu's (1971) modification of Palmgren's (1960) silver stain to determine the presence or the absence of nerves in the segments of the limbs under investigation. Skeletal preparations (cleared and stained with alizarin red and toluidine blue) were made from some older specimens.

\section{RESULTS}

\section{Series 1. Simple Joining of Forearm.s}

The operative model consisted of the basic limb-joining procedure described above. The limbs remained joined throughout the experiment. Thirty fused limbs, fixed mainly during the first month after joining, were examined histologically in order to ascertain the nature of the healing reactions along the plane of fusion. Seventeen limbs were followed for long periods (up to 11 months), and any gross regenerative changes were recorded.

The early histological events following fusion of the limbs were unremarkable. Within 24 hours epithelial continuity had been established between most fused limbs. In some regenerates, epithelial ingrowths partially separated the opposed mesodermal tissues during the first week. but during later periods the epithelium retracted toward the surface. The stage of phagocytosis and demolition did not differ from that which occurs in postamputational limb regeneration. Beginning early in the third week, osteoclasts, in small numbers, attacked the ends of the bones. The extent of osteoclastic activity, however, was less than that seen in the amputated limb (Fig. 1). The dedifferentiative reaction of the soft tissues, particularly muscle, was sharply reduced. Although a limited amount of dedifferentiative activ- 


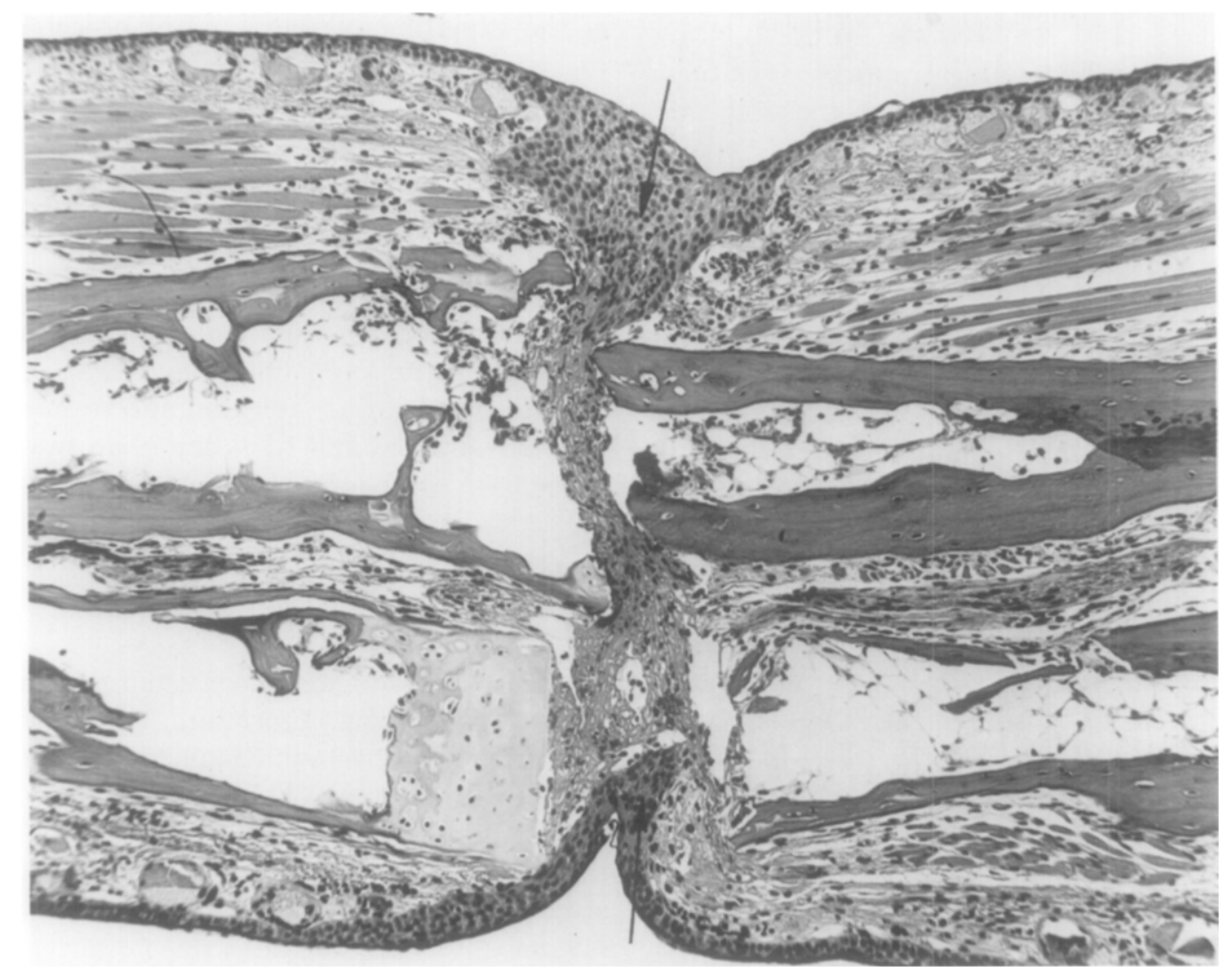

FIG. 1. Longitudinal section showing the area of limb fusion 18 days postoperatively. The radii and ulnae are well aligned and have undergone slight osteoclastic erosion. Some ingrowth of epidermis (arrows) is present at the site of fusion. The fusion plane itself is occupied by loose tissue containing little collagen. H \& E. $\times 48$.

ity occurred in some of the peripheral muscle bundles associated with the wound epithelium, large masses of intact muscles were seen at the plane of fusion during the fourth week, or later, after limb fusion (Fig. 2). Normally in an amputated $\operatorname{limb}$, the dedifferentiative reaction has removed almost all traces of intact muscle fibers from the last millimeter of the limb stump by the end of the third week. Connective tissue staining revealed that remarkably little collagenous tissue was laid down at the plane of limb fusion.

Of the 17 fused limbs which were followed grossly for long periods, two remained morphologically stable. Hypomorphic regenerates appeared at the site of fusion on the remaining 15 limbs. Eight of the regenerates were single one- or twodigit spikes occurring on the anterior or posterior face of the fusion line (Fig. 3). The other 7 fused limbs produced double regenerates (Fig. 4). These were also spike regenerates, and were consistently seen on opposite sides of the fusion surface.

\section{Series 2. Denervation of One Arm and Joining of Forearms}

It is known that for normal limb regeneration three conditions must be met: (1) a wound epithelium not underlain by dermis or a basement membrane, (2) mesodermal damage, and (3) a quantitatively adequate nerve supply (reviewed by Carlson, 1974). From the standard histological examination of fused limbs in Series 1, the 


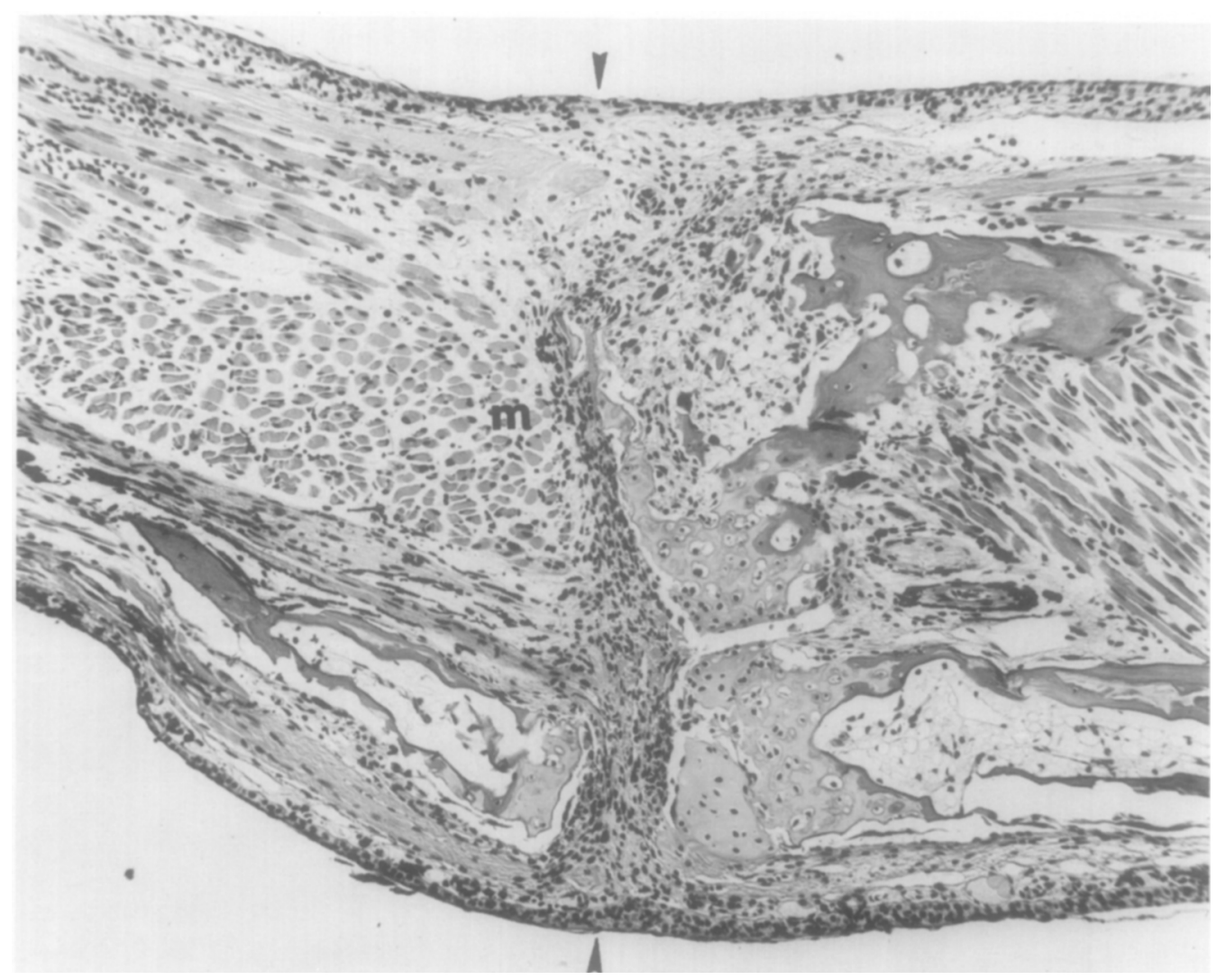

FIG. 2. Longitudinal section through joined limbs 21 days after fusion. Large numbers of essentially intact muscle fibers $(m)$ are present next to the fusion plane (arrows) in contrast to the extensive dedifferentiative reaction that would normally occur in an amputated limb covered with a wound epithelium. The transected bones show prominent osteoclastic activity. H \& E. $\times 42$.

presence of a wound epithelium and damaged mesoderm were verified. Because regeneration occurred, it was assumed that a quantitatively adequate number of nerve terminations was also present in or near the epidermis at the site of limb fusion.

This experiment was designed to find out whether the absence of intact nerves in one of the fused limbs would permit nerve fibers regenerating from the other limb to pass through the site of joining. In 20 newts the right arm was denervated by severing spinal nerves 3,4 , and 5 along the lateral body wall. Because denervation effectively immobilized the right arms, a portion of the humerus was removed from the left arms only. Both forearms were amputated through the distal third and fused as described above. The limbs were allowed to remain fused throughout the duration of the experiment. To ensure that the right arms remained free of nerves throughout the early phases of the experiment, repeated denervations of the right arms were performed 15 and 30 days after the day of fusion of the limbs. After the third nerve resection further accurate denervations were not possible because of the scarring which obscured the nerve fibers.

Thirteen animals were followed for 90 days. In no case did supernumerary limbs form at the site of joining of the limbs. 


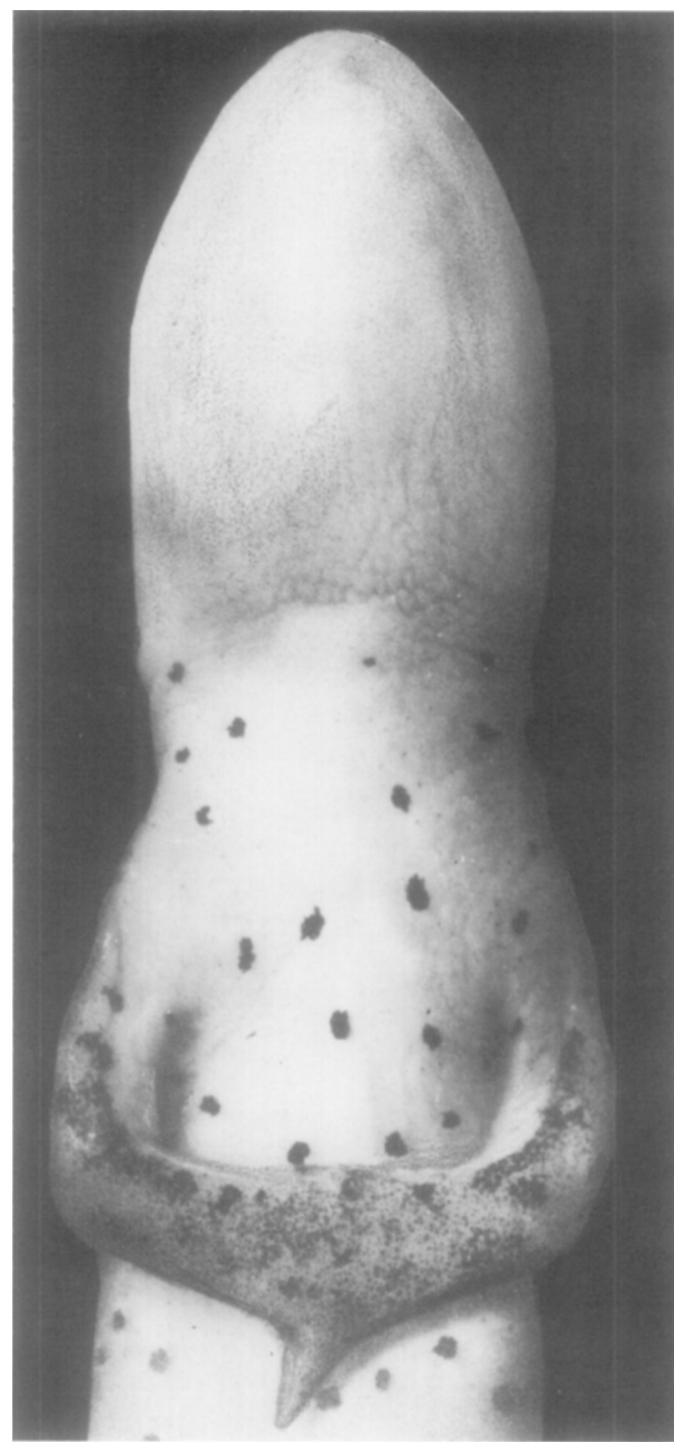

FIG. 3. Single-spike regenerate growing out from the fusion plane 266 days after the limb-joining operation.

Series 3. Joining of Forearms and Subsequent Amputation of One Upper Arm at the Shoulder

The basic forearm fusion operation was performed as described in Materials and Methods in 25 newts. After the limb joining operation, the newts were placed in the cold to allow soft tissue healing as well as cross-anastomoses of the blood vessels to occur. Initially, the limbs remained fused for periods of 15-18 days before the right upper arm was amputated at shoulder level. The terminal portion of the amputated fused limb was of reversed polarity, the proximal part of the right upper arm being the apical end. In later animals, amputation was performed at intervals of 6-10 days after joining. The reaction of the fused limbs to amputation was closely related to the time interval between limb fusion and amputation. Limbs which were

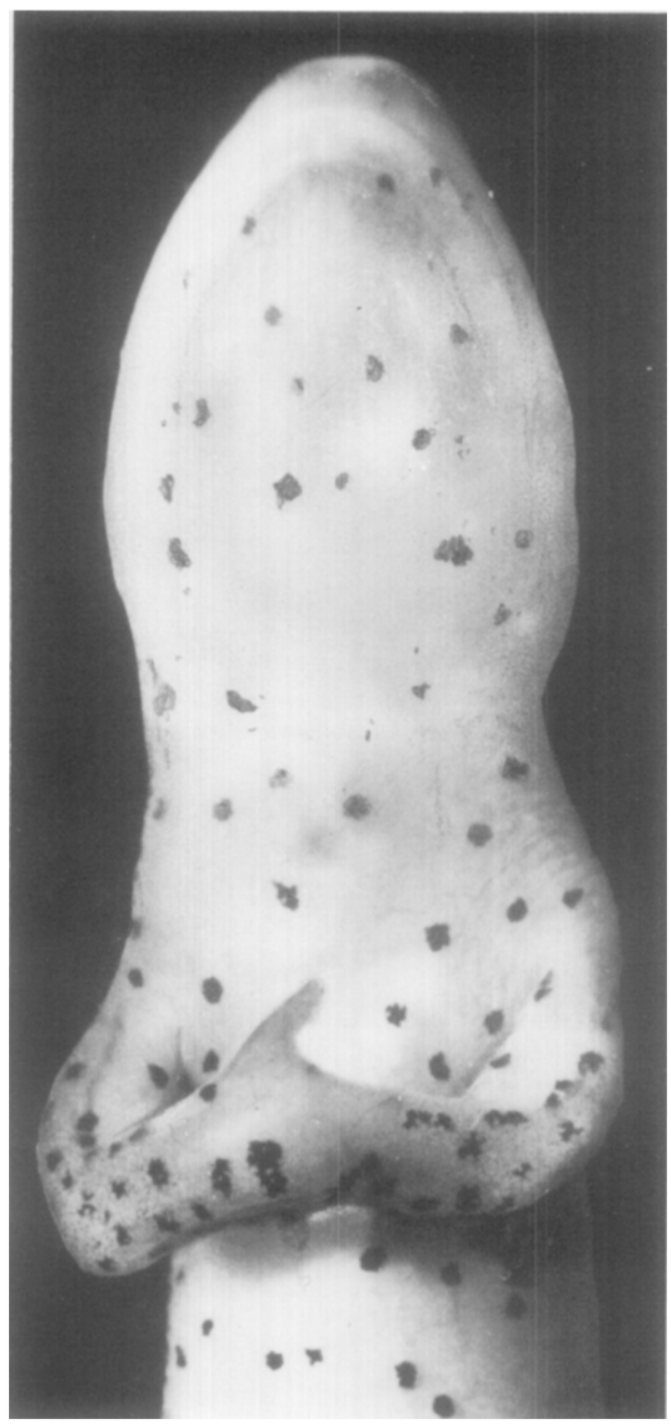

FIG. 4. Two-spike regenerates growing approximately $180^{\circ}$ apart at the plane of fusion. 322 days postoperatively. 
amputated more than 9 days after joining underwent healing and remained morphologically stable. Regeneration did not occur in these limbs. Fused limbs which had been joined for 6-8 days prior to amputation remained joined, but regression of the terminal part of the fused right arm was the rule. The extent of regression in the reversed right arms varied considerably. In mild cases the reversed upper arm segment regressed almost to the elbow. In more severe cases regression continued through the elbow into the forearm. All limbs which underwent regression eventually formed blastemas and regenerated morphologically normal arms and hands (Fig. 5). As is the rule in regenerates arising from $\operatorname{limb}$ stumps with reversed polarity, the regenerated arms displayed a right-left symmetry opposite to that of the limb which formed the amputation surface. Thus, in this case left limbs arose from the reversed right limb stumps.

The nonregenerating limbs which had been amputated 9-18 days after joining were reamputated 30 days or more after the first amputation when it became apparent that healing had been completed and that the morphology of the limbs was stable. In all cases the reamputated limbs produced regenerates with left symmetry (Fig. 6).

Sixteen animals in this series were fol

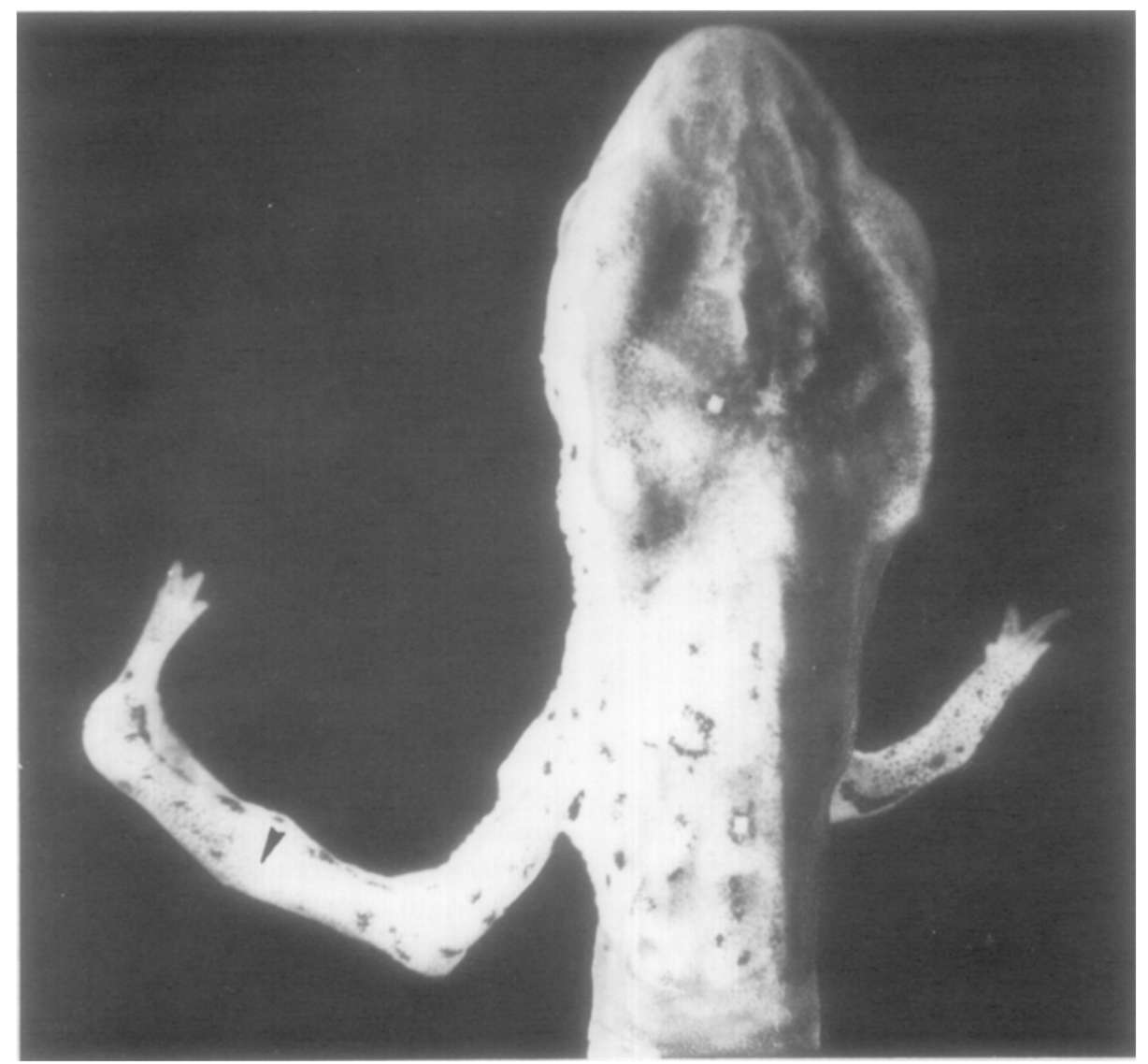

Fig. 5. Very small but morphologically complete regenerate growing from a fused arm that had regressed to the elbow. The plane of fusion is indicated by an arrow. The contralateral limb is a normal regenerate arising from the shoulder after separation of the fused limbs. Arms fused for 8 days before amputation. One hundred eleven days after amputation. 


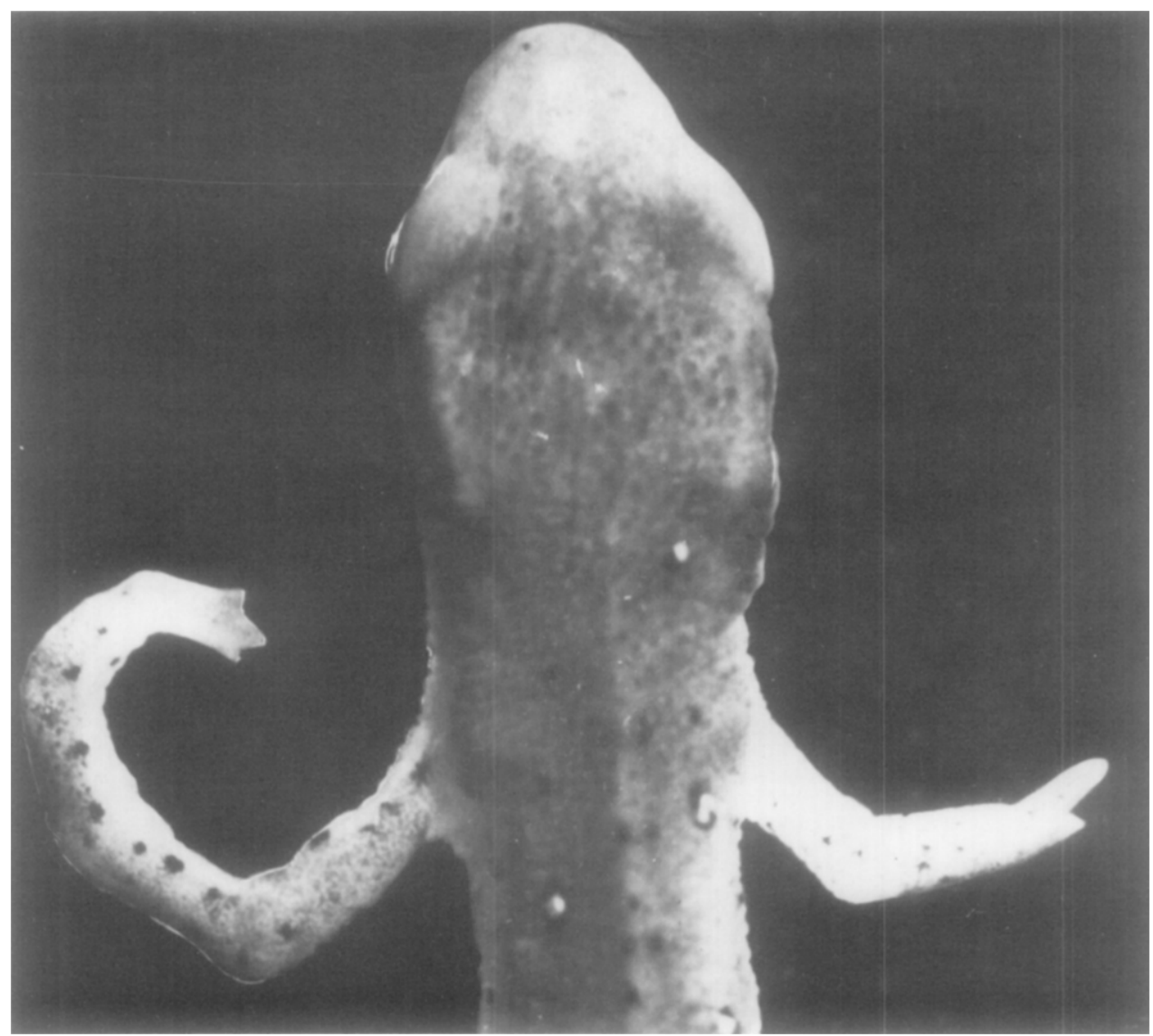

FIG. 6. Forearms fused for 19 days before amputation. No regeneration followed, and the limb was reamputated 1 month later. A regenerate with left symmetry, possessing part of an upper arm segment and all structures distal to the elbow, has arisen from the amputation surface of the reversed right arm.

lowed for a long term (up to 12 months). Of these limbs, only one formed a supernumerary structure at the site of limb fusion in addition to a terminal regenerate (Fig. 7).

Series 4. Denervation of One Arm, Joining of Forearms, and Subsequent Amputation through the Denervated Arm

The lack of terminal regeneration following the first amputation in nonregressing limbs of Series 3 suggested that immediately after amputation the terminal right limb segment was denervated and that the amputation surface became blocked off by connective tissue before regeneration could occur. As a test of this possibility a varia- tion of the procedure in Series 3 was performed. In 30 newts the right arm was denervated as in Series 2, and a portion of the left humerus was removed. Both forearms were amputated and joined in the usual manner. All right arms were amputated at the shoulder 15 days after the limbs were joined. In series 3, neither regression nor regeneration occurred in terminal right arm segments amputated this late after fusion. Of the long-term animals in the present series, all produced regenerates after the first amputation. The morphology of the regenerates did not differ from those which formed in Series 3 . No regeneration of supernumerary structures occurred at the sites of limb fusion. A high 


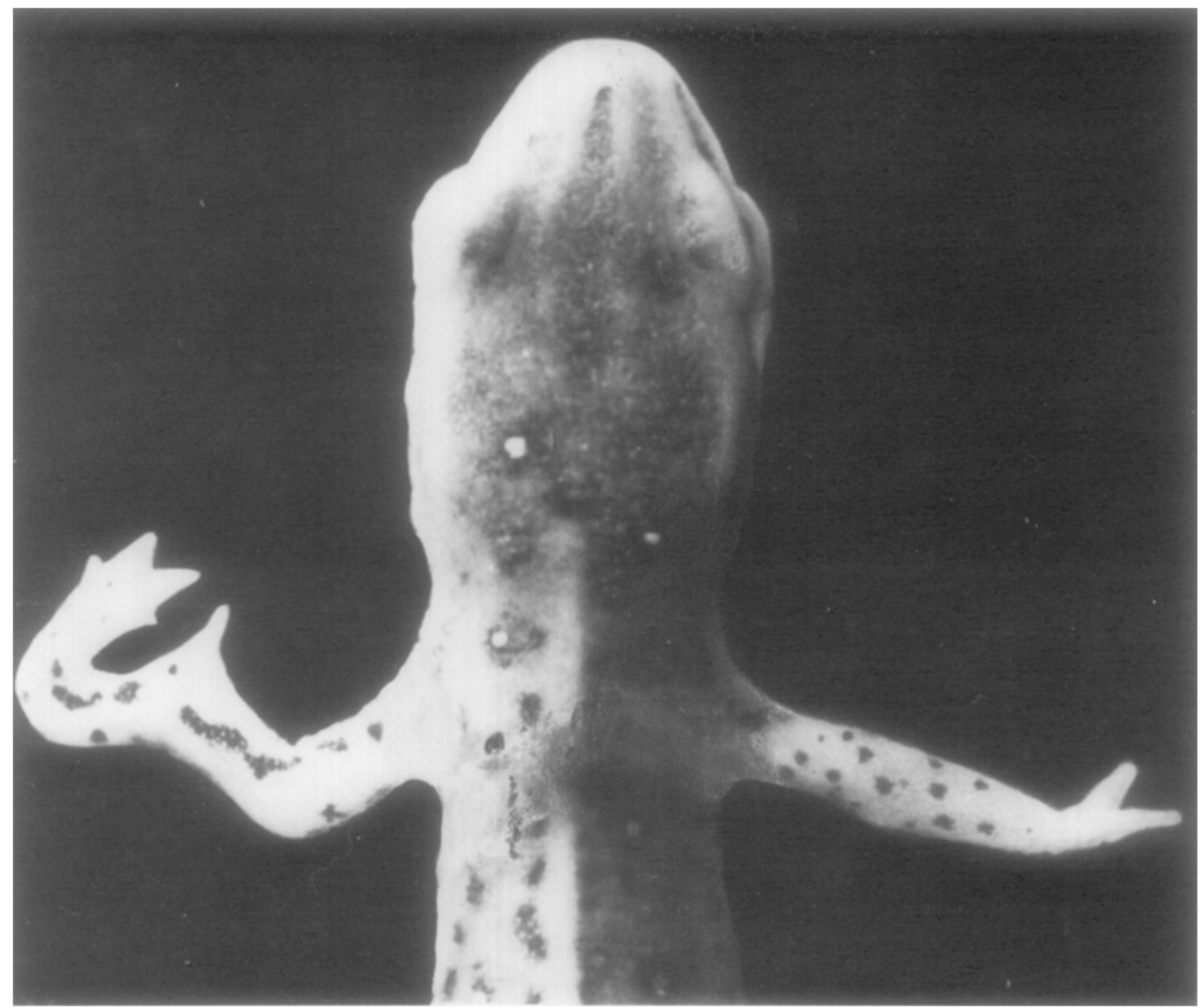

Fic. 7. Forearms fused for 10 days before amputation of right arm at shoulder. This was the only case in which a supernumerary spike regenerate appeared at the point of fusion in addition to a new regenerate forming at the amputation surface. 108 days after amputation.

percentage of the regenerates which appeared in both Series 3 and 4, even though morphologically normal, were considerably smaller than normal regenerates of the same age (Figs. 5 and 8 ).

\section{Nerve Staining Results}

In addition to the work reported above, silver staining for nerves was performed on numerous other limbs in these experimental series in order to substantiate some of the conclusions derived from gross observations of the regenerative phenomena reported above. This work was designed to determine primarily the presence or the absence of nerve fibers in various regions at selected times rather than to test directly the overlapping or repulsion of regenerating nerves. The latter problem is the sub ject of a separate study now in progress.

Simple nerve staining did not shed much light on Series 1. Nerve fibers do innervate the wound epidermis at the plane of junction, and there is some evidence that occasionally outgrowing fibers from opposing nerves may innervate the same area of wound epidermis or the same focus of regeneration. In other cases major opposing nerve trunks were seen to supply different regenerates. On the basis of staining simply fused limbs in this series. nothing definitive could be offered on the question of nerve interactions.

In Series 2 silver staining revealed 


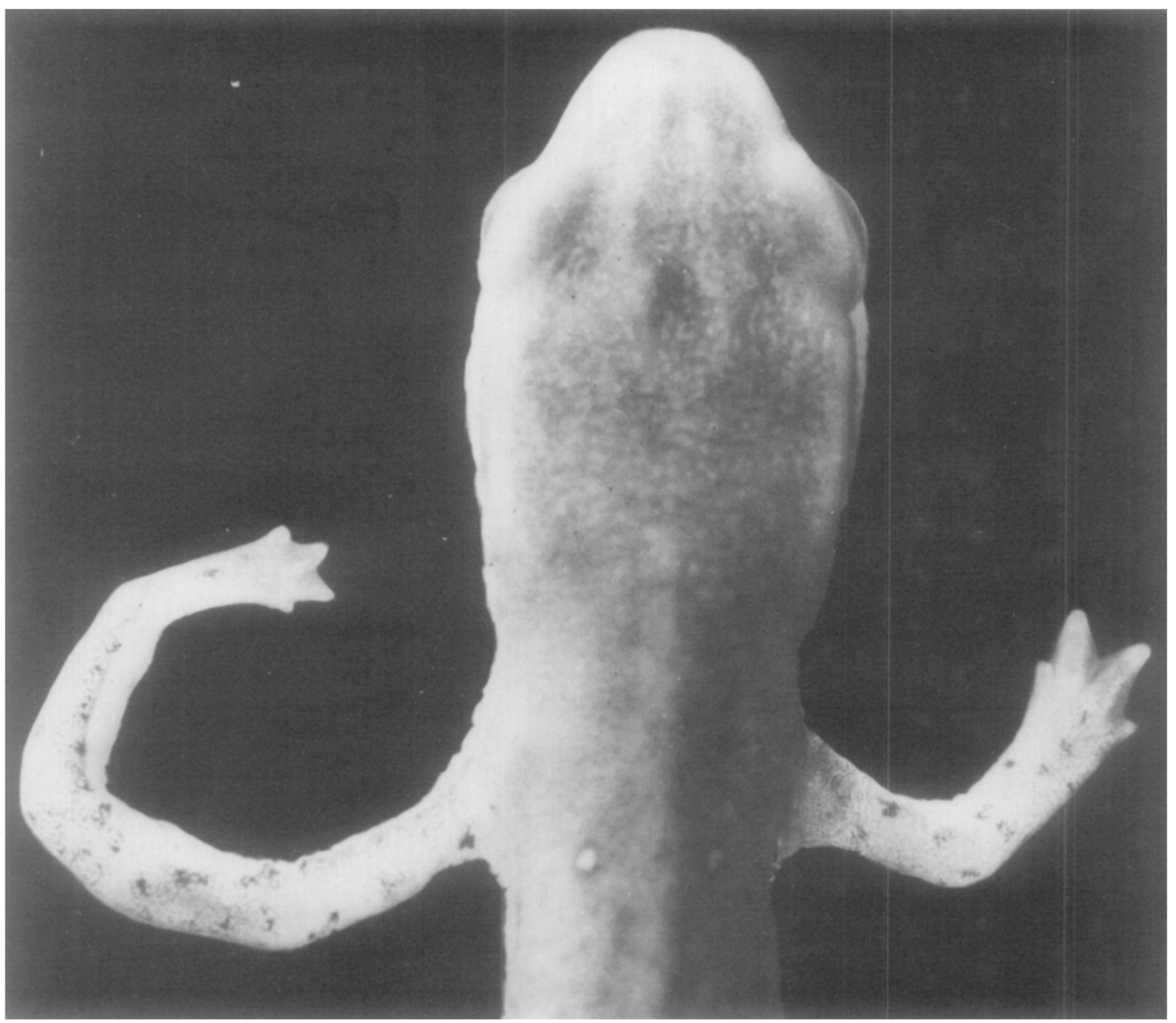

Fig. 8. Right arm denervated and forearms fused for 15 days prior to amputation of right arm at shoulder. Ninety-six-day regenerate. Note the difference in size between the left and right regenerates. Both are of the same age.

unquestioned evidence of nerve ingrowth from the normally innervated limb across the line of fusion into the denervated one. By 5-7 days regenerating nerve fibers were seen at the fusion plane, and by $8-9$ days the first evidence of nerve crossover was obtained. At later periods larger bundles of nerve fibers penetrate the contralateral denervated limb (Fig. 9). Branching from these bundles may be small numbers of fibers which terminate in the wound epidermis of the junctional area. The regenerating nerves progress proximad primarily through the deep tissues of the contralateral limb and reach the elbow area by the third week after denervation and fusion. In one 23-day animal regenerating muscle fibers, in addition to nerves, were seen crossing the plane of fusion into the denervated limb (Fig. 10).

The amputated limb in Series 3 is denervated from the site of fusion to the amputation surface at the time of the first amputation (Fig. 11). By the time connective tissue begins to form around the end of the amputated fused limb, nerves from the contralateral limb have not yet penetrated as far as the amputation surface. However 1 month after amputation (the time of reamputation) large nerve trunks are seen at the end of the amputated arm. Regenerates arising after the second amputation are innervated. Gross testing revealed both sensory and motor function. In addition, 


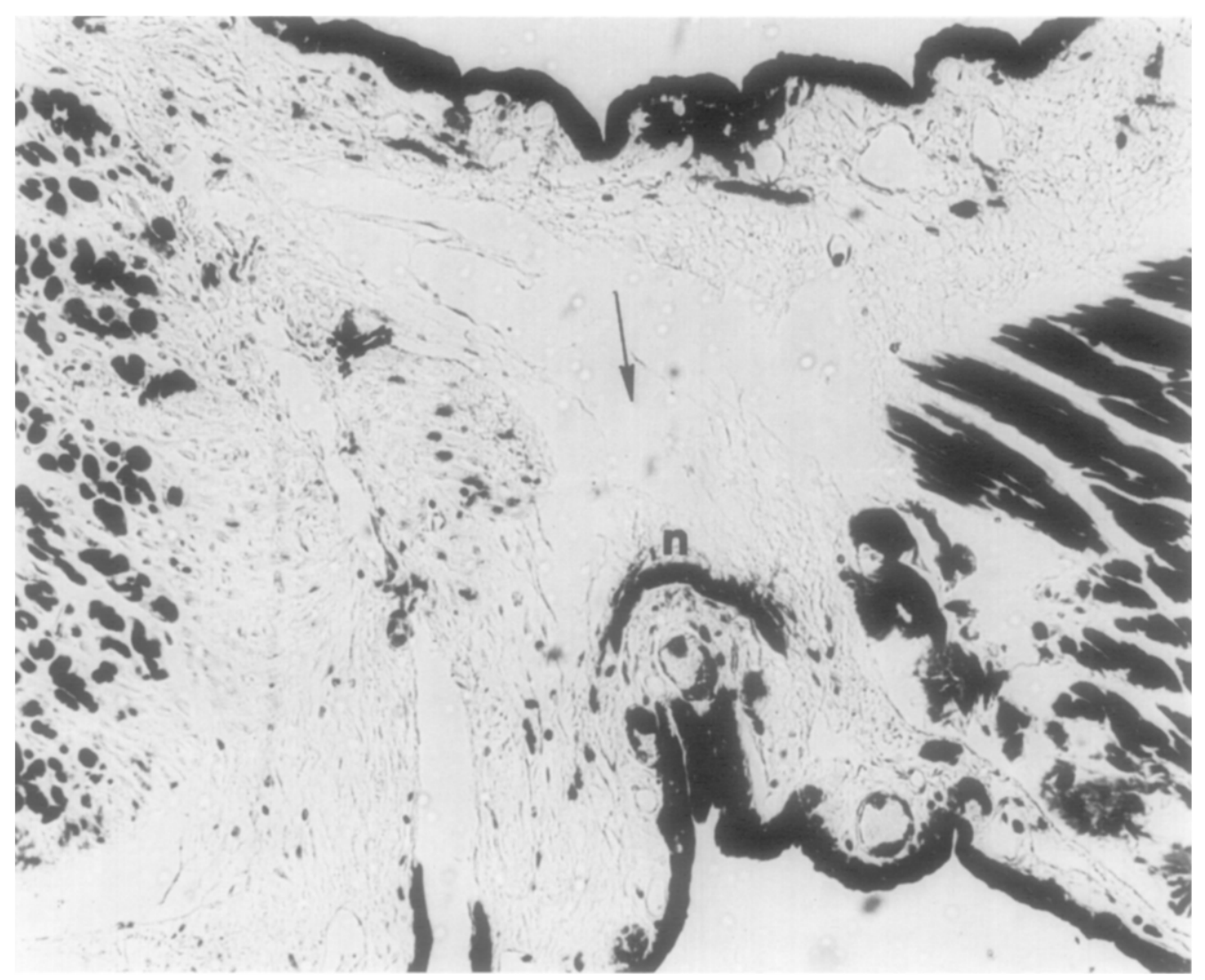

Fig. 9. Section through the plane of fusion in a 27-day postoperative animal in Series 2. A prominent nerve trunk $(n)$ traverses the plane of fusion (arrow) into the denervated limb (left). Palmgren's stain. $\times 82$.

silver-stained cross sections of regenerates demonstrated the presence of nerve fibers.

The results of nerve-staining in Series 2 serve also to demonstrate that in Series 4 when the originally denervated limb is amputated 15 days after fusion, a plentiful nerve supply has already grown in from the contralateral limb. Nerve fibers which had not yet reached the plane of amputation would have up to a week to continue regenerating distad hefore the amputation surface would begin to become sealed off by fibrous tissue.

\section{DISCUSSION}

The results of these experiments shed some light upon a number of problems related to the regeneration of both peripheral nerves and limbs. The primary ques- tion which instigated this investigation was how regenerating nerves of opposite polarity would react when their terminals meet. Would they ignore the contralateral nerve and continue to advance past one another, or would they exert a mutually exclusive effect and cease to advance? Fven though the experiments included in this report were not initially expected to provide the answer to this question, it is possible to say with reasonable assurance that a regenerating peripheral nerve, at least in this system, will not regenerate to a significant extent into a territory already occupied by a nerve of opposite polarity. The evidence supporting this conclusion is primarily indirect and is based upon our knowledge of the relationship between nerves and limb regeneration. The appearance of an epi- 


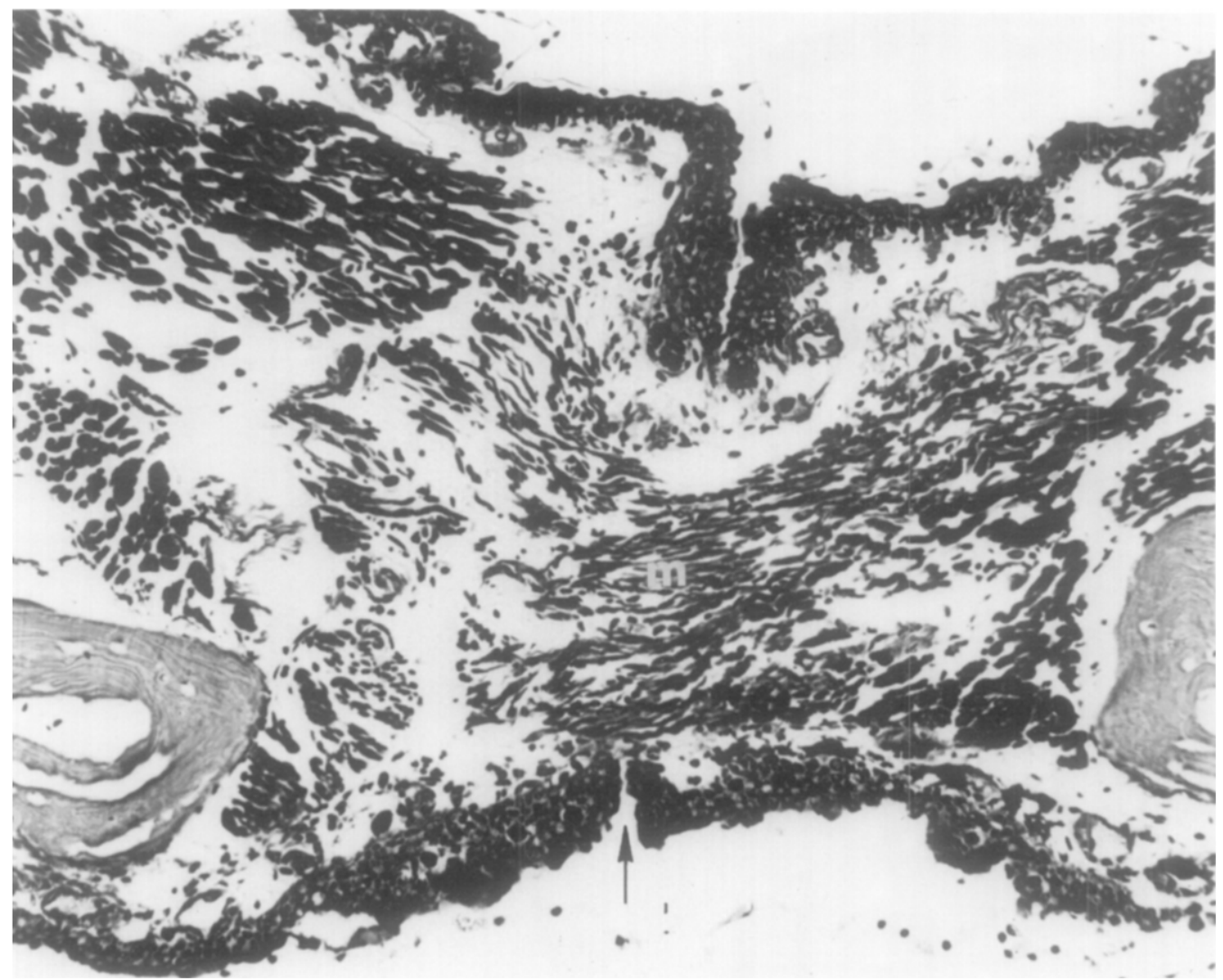

Fig. 10. Section of 23-day postfusion limbs demonstrating the regeneration of muscle fibers $(m)$ across the plane of fusion (arrow) and into the denervated limb (left). Palmgren's silver stain. $\times 80$.

morphic regenerative response was used as a gross morphological indication that nerve fibers were present at the cut end of a limb at the time of amputation or in the early wound epidermis at the plane of joining between two fused limbs. Conversely, the absence of regeneration was interpreted to mean that nerve fibers in adequate number were not present at the right place or time to support regeneration. The gross regenerative results and the distribution of nerves that would account for them are summarized in Fig. 11. The conclusion that a regnerating nerve will not make a substantial penetration into the territory occupied by its contralateral counterpart is supported by the following evidence:

I. Evidence for the exclusion of regenerating nerve fibers from territory occupied by nerves of opposite polarity. (1) The high percentage of regeneration of spikelike structures in Series 1. This type of regneration does not occur in the absence of nerves terminating within or in the vicinity of the wound epidermis, and its presence in this experiment suggests that the regenerating nerve fibers terminate in the area of the epidermis at the site of fusion, a fact later confirmed by specific nerve stains. (2) In Series 3 , nonregressing limbs did not regenerate after the first amputation, but all regenerated after a second amputation. This result is interpreted to mean that at the time of the first amputation nerves from the left arm had not crossed into the right arm. Thus shortly after amputation the inverted right arm segments contained only degenerating right nerves and were not able to support regeneration before scar tissue sealed off the amputation surface. In 

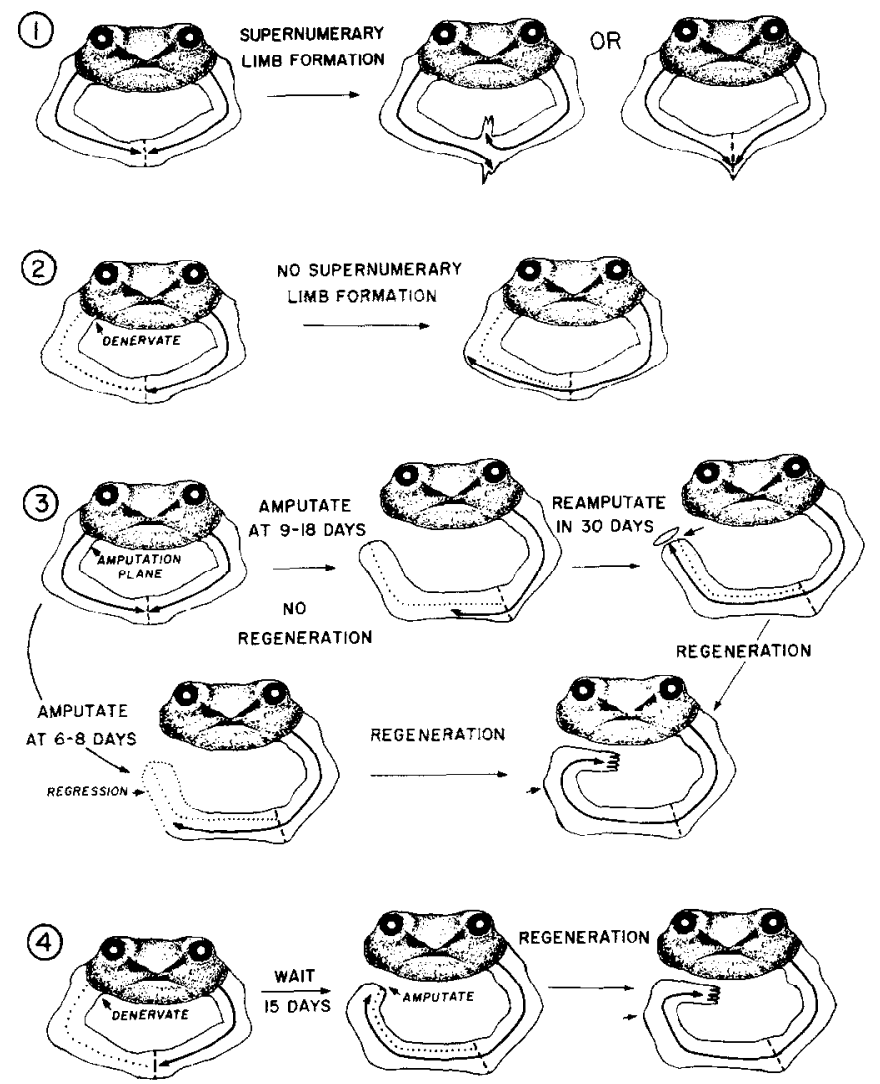

FIG. 11. Diagram summarizing the operative procedure, the gross regenerative results, and the hypothesized nerve interactions in Series 1-4. Intact and regenerating nerves are represented by the heavy line ending with an arrow. Degenerating nerves are represented by dotted lines. Arrows perpendicular to the limbs refer to amputation sites.

the time between the first and second amputation of the inverted right arm, nerves from the left limb would have had time to regenerate to the end, so that by the second amputation all the conditions necessary for limb regeneration were met. The presence of nerve trunks at the ends of the limb stumps in Series 3 at the time of the second amputation was verified by silver staining. That these terminal regenerates were, indeed, innervated was ascertained both by direct observations of their motor ability and responses to sensory stimulation and by cross sectional analysis of silver-stained regenerates.

II. Evidence in favor of nerve ingrowth into the denervated contralateral fused limb. (1) In Series 2, no spikelike struc- tures regenerated at the site of limb fusion. This indicated that fibers of the regenerating left nerves, instead of remaining in the area of limb fusion, proceeded directly into the denervated right limb. In this case, either no nerve terminations remained at the site of limb fusion or if any did remain. their number was insufficient to support a regenerative response. (2) Regeneration of a limb after the first amputation in Series 4 means that an intact nerve supply was present at the plane of amputation of the initially denervated right limb. This could only be accomplished by the ingrowth of ncrves from the left limb fused to it. (3) The lack of regeneration at the site of limb fusion in 27 of 28 animals in Series 3 and 4. This is interpreted to mean that in the 
fusion followed-by-amputation experiments the regenerating left nerve bypassed the wound epithelium at the site of limb fusion in a manner similar to that which occurred in Series 2. The one case of regeneration at the site of $\operatorname{limb}$ fusion in amputated limbs occurred in Series 3. In this series, two nerves of opposite polarity confronted one another until the time of amputation (6-19 days). Undoubtedly during this interval nerves had begun to penetrate the wound epidermis as occurred in Series 1. It is therefore surprising that more supernumerary regenerates did not form at the site of fusion in Series 3. Again using the gross results as a point of departure, one can surmise that early nerve-epithelial approximations were made at the site of fusion, but that when the influence of the right nerves was withdrawn by amputation, the left nerve terminals took advantage of the vacuum and became redirected into the right limb. This has not yet been tested by direct observation or experimentation.

The method of analysis used above, concentrating upon gross observation of regenerating limbs, has some obvious limitations. One cannot conclude that there is no overlap of regenerating nerve fibers in areas of confrontation of nerves with opposite polarities or that all nerve fibers from an innervated limb enter the fused denervated limb segment. Nevertheless, ongoing analysis of the problem in this laboratory attests to the essential correctness of the conclusions arrived at by the approach used in this report.

It is well recognized that in areas where tissues are approximated in such a way that their polarities are not in phase, morphological instability is common (see summaries in Korschelt, 1927, and Rose, 1970). This phenomenon has recently been demonstrated with respect to the dorsoventral and anteroposterior limb axes by Bohn (1965) and Bullière (1970) in insects and by Droin (1959), Settles (1967), and Carlson
(1972) in urodele amphibians. Experimental confrontations of tissues with opposite proximodistal polarity, however, have been attempted considerably less frequently. Despite expectations to the contrary, there were no histological abnormalities in the course of epidermal wound healing at the site of fusion in these experiments. The regenerative responses seen in Series 1 may have been facilitated by tissue instability at the plane of fusion, but in that case one would have to assume that opposing nerves were largely responsible for the instability, even if their influence was mediated through other tissues. Otherwise one would expect at least some regeneration to have occurred in Series 2.

One of the striking histological phenomena in the fused limb series (Series 1) was the great reduction in the extent of the dedifferentiative reaction of the soft tissues along the plane of fusion. Normally in this laboratory, early signs of dedifferentiation can be seen at $10-11$ days. The height of the dedifferentiative process occurs early during the third postamputational week, with blastema formation beginning at the end of the third week. In the case of fused limbs, some dedifferentiation takes place in the superficial muscle and connnective tissue layers, but often original muscle fibers remain intact in the central areas along the fusion line for 3 weeks or more (cf. Fig. 2). Of course in areas where spike regenerates form, substantial amounts of local dedifferentiation are seen. The presence or absence of dedifferentiation in the fused limbs is roughly correlated with the presence or absence of a wound epidermis. Variations in the amount of dedifferentiation appear to be related to the extent of penetration of epidermis at the plane of fusion. Throughout the years there have been suggestions that in adult urodeles contact with a wound epidermis is necessary to initiate or facilitate the dedifferentiative response (Carlson, 1974), but most of the evidence has been indirect. In 
the present work one can say that the findings are suggestive of, but by no means constitute definitive proof for, such an action of the wound epidermis.

The results of Series 3 and 4 provide definitive proof that the ability to start regenerating or the signal to stop regenerating in a limb is not directly related to the absolute distance of the regenerating structure from the midline of the body. This was already demonstrated by Dent (1954), who reported the regeneration of newt limbs with reversed polarity. The reversed limbs were implanted into the body wall. In these reversed limbs, the plane of amputation was still no farther from the midline than the length of a normal amputated limb, but the regenerates terminated at a distance farther from the midline than normal regenerates. In Series 3 and 4 of this experiment the plane of amputation was farther from the midline than the end of a normal limb, and the regenerates terminated at a length over 2.5 times that of a normal limb.

In addition to producing regenerates at abnormally long distances from the midline, the operations in Series 2, 3, and 4 are methods by which the length of a peripheral nerve can be greatly elongated. These techniques of nerve lengthening could be useful for producing limb nerves long enough for experimental manipulations involving the supplementary innervation of contralateral limbs or other areas in the body by means of surgical deviation of nerves.

$\Lambda \mathrm{ll}$ regenerates in Series 3 and 4 were of left symmetry even though they arose from reversed right limbs. This confirms the prior reports of this phenomenon in regenerating urodele limbs (Butler, 1951; Deck, 1955; Dent, 1954).

The often extensive regression of the right limb segments amputated short periods after fusion with the left (Series 3 ) is worthy of comment. In contrast to Ambystoma larvae, regression is rare in amputated limbs of newts. Rose (1948) remarked that he did not see regression in denervated limbs of adult newts after amputation. In Series 3 two factors, denervation and poor vascularization, are possible causes of regression. The evidence points to poor vascularization as the likely cause because none of the fused right limbs in this series were innervated just after amputation. Yet the limbs which had been fused for over 10 days did not regress following amputation. Had lack of innervation been responsible for regression, these limbs should have regressed also. It appears that limbs fused for only 6-8 days prior to amputation had not developed sufficient vascular anastomoses to maintain the terminal portion of the fused right limbs after their main blood supply had been cut off.

The regressing limbs of Series 3 eventually regenerated after the first amputation. It is known that in a regressing limb a cap of dense scar tissue does not form over the wound surface and that when nerves reach the apical epidermis the regression becomes stabilized and regeneration ensues (Thornton and Kraemer, 1951). In Series 3, regression of the fused right limb segment probably continued until vascular connections and ingrowing nerve fibers from the left nerves created conditions amenable to a progressive regenerative response.

Finally, the pattern of regeneration of the fused limbs in Series 1 can be examined in the light of interpretations of other limb fusion experiments. In cases reported in the literature (Monroy, 1946; Goss, 1956; Oberpriller, 1968), the forearms were fused in a parallel fashion so that the proximodistal axes tended to converge rather than directly oppose one another as was the case in Series 1. These reports described regenerates which usually started out double at the base and then converged at their apex. The results were intrepreted on the basis of convergence of morphogenetic fields.

In Series 1, 7 of 15 fused limbs produced two regenerates developing from separate 
foci. They normally arose close to $180^{\circ}$ apart along the plane of limb fusion. Thus these regenerates do not fit the anatomical or theoretical pattern which has been common to the other limb fusion experiments. It seems likely that each of these regenerates is innervated separately. Their consistent appearance on opposite sides of the plane of fusion may be a further reflection of nerve repulsion. Several of the single regenerates in this series arose on the inside of the " $V$ " if the fused limbs became fixed at slight angles $\left(150^{\circ}-170^{\circ}\right)$ instead of a straight line. This again points to the location of regenerates being determined by random factors, such as the availability of a terminal nerve supply rather than being strictly controlled by polarized linear forces emanating through and out of the limb stump. Their poor development, however, could be interpreted on the basis of cancellation of conflicting streams of morphogenetic information. On the other hand, it could also be merely a reflection of an insufficient number of cells comprising the regeneration blastema.

The authors wish to thank Mrs. Sherry Rogers for technical assistance and Mrs. Ethel Abrahams for typing. Gross photography was done by Mr. William Brudon.

\section{REFERENCES}

Boнs, Н. (1965). Analyse der Regenerationsfähigkeit der Insektenextremität durch Amputations und Transplantationsversuche an Larven der afrikanischen Schabe (Leucophaea maderae Fabr.). Wilhelm Roux Arch. Entwicklungsmech. Organismen 156, 449-503.

Bullière, D. (1970). Interprétation des régénérats multiples chez les Insectes. J. Embryol. Exp. Morphol. 23, 337-357.

Butler, E. G. (1951). The mechanics of blastema formation and regeneration in urodele limbs of reversed polarity. Trans. N. Y. Acad. Sci. 13, 164-167.
Carlson, B. M. (1972). Morphogenetic interactions between skin and underlying tissues during limb regeneration. Amer. Zool. 12, 703 (Abstract).

Carlson, B. M. (1974). Factors controlling the initiation and cessation of early events in the regenerative process. In "Neoplasia and Cell Differentiation" (G. V. Sherbet, ed.), pp. 60-105. Karger, Basel.

DEck, J. D. (1955). The innervation of urodele limbs of reversed proximo-distal polarity. J. Morphol. 96, 301-331.

DENT, J. N. (1954). A study of regenerates emanating from limb transplants with reversed proximodistal polarity in the adult newt. Anat. Rec. 118, 841-856.

Droln, A. (1959). Potentialités morphogènes dans la peau du Triton en régénération. Rev. Suisse Zool. 66, 641-709.

Goss, R. J. (1956). The unification of regenerates from symmetrically duplicated forelimbs. J. Exp. Zool. 133, 191-209.

Hsu, L. (1971). The role of nerves in the regeneration of minced muscle in adult anurans. Ph.D. Thesis, Univ. of Michigan, Ann Arbor, 109 pp.

KoRsChelt, E. (1927). "Regeneration und Transplantation," I. Band: Regeneration. Borntraeger, Berlin.

Monroy, A. (1946). Richerche sulle rigenerazione degli Anfibi urodeli. Nota III. Osservazioni su rigenerati formati si su doppie superfici ai sezione e considerazioni sui processi determinativi della rigenerazione. Arch. Zool. Ital. 31, 151-172.

Oberpriller, J. (1968). The action of X-irradiation on the regeneration field of the forelimb of the adult newt, Diemictylus viridescens. J. Exp. Zool. 168, 403-422.

Palmgren, A. (1960). Specific silver staining of nerve fibers. I. Technique for vertebrates. Acta Zool. 41, 239-265.

Rose, S. M. (1948). The role of nerves in amphibian limb regeneration. Ann. N. Y. Acad. Sci. 49, 818-833.

Rose, S. M. (1970) . "Regeneration." Appleton-Century-Crofts, New York.

Settles, H. E. (1967). Supernumerary regeneration caused by ninety degree skin rotation in the adult newt, Triturus viridescens. Thesis, Tulane Univ., New Orleans, Louisiana.

Thornton, C. S., and Kraemer, D. W. (1951). The effect of injury on denervated unamputated fore limbs of Amblystoma larvae, J. Exp. Zool. 117, $415-440$. 